HAIT Journal of Science and Engineering A, Volume 5, Issues 1-2, pp. 265-272

Copyright (C) 2008 Holon Institute of Technology

\title{
Is there a linewidth theory for semiconductor lasers?
}

\author{
Boris Spivak ${ }^{1}$ and Serge Luryi ${ }^{2, *}$ \\ ${ }^{1}$ Dept. of Physics, University of Washington, Seattle, WA, 98195, USA \\ ${ }^{2}$ Dept. of Electrical and Computer Engineering, \\ University at Stony Brook, NY, 11794-2350, USA \\ *Corresponding author: Serge.Luryi@stonybrook.edu
}

Received 8 September 2007

\begin{abstract}
Laser generation begins at a critical injection when the gain and loss spectra touch each other at a singular frequency. In the framework of the standard theory, the finite linewidth results from the account of fluctuations associated with the random spontaneous emission processes. This approach is based on the assumption that in the meanfield approximation the singular frequency generation persists for injection levels higher than critical. We show that this assumption in the framework of the Boltzmann kinetic equation for electrons and photons is invalid and therefore the standard description of semiconductor laser linewidth lacks theoretical foundation. Experimental support of the standard theory is also questionable.
\end{abstract}

PACS: 42.55.Ah, 42.55.Rx, 78.40.Fy

\section{Introduction}

Laser linewidth theory was pioneered by Schawlow and Townes [1] and further developed in [2] and [3]. We discuss the status of the Schawlow-TownesLax-Henry (STLH) theory of laser linewidth in the instance of semiconductor injection lasers. At injection levels $I$ below threshold $I<I_{C}$ one can introduce two spectra $g(\omega, I)$ and $\sigma(\omega)$, describing, respectively, the material gain 
and the loss at cavity mirrors of the electromagnetic field intensity. The gain $g(\omega, I)$ is generally an increasing function of $I$. At $I=I_{C}$ the two spectra touch each other, $g\left(\omega_{0}, I_{C}\right)=\sigma\left(\omega_{0}\right)$, and the generation begins. The STLH theory of laser linewidth is based on the assumption that in the mean-field approximation (i.e., without fluctuations) the singular in frequency nature of generation persists above the threshold (i.e., for $I>I_{C}$ ). In the framework of this approach, the laser line acquires a finite width $\Gamma$ entirely due to fluctuations. In an ideal laser these fluctuations are due to the random discrete nature of spontaneous emission.

We shall refer to the property of the two spectral curves $g(\omega, I)$ and $\sigma(\omega)$ to touch each other at a singular frequency for $I>I_{C}$ as rigidity. In principle, however, scenarios other than rigidity are also possible. For example, the curves may touch each other for $I>I_{C}$ in a finite interval of frequencies, so that there is a finite linewidth even in the mean-field approximation. In this case, the account of fluctuations would provide only a correction. This is not an unusual situation. For example, the conventional mean-field scenario for multimode laser generation involves oscillations at several discrete frequencies.

In this paper we examine the validity of the assumption of rigidity. First we briefly review the standard STLH linewidth theory. Next we derive a mean-field expression for the linewidth using Boltzmann's kinetic equation for electrons and photons. In this approach the linewidth turns out to be an increasing function of injection, which violates the assumption of rigidity and is in contradiction with the STLH scenario. Curiously, however, it is not necessarily in contradiction with experiment.

The simplest model of the laser is a pumped two-level electronic system, immersed in an electromagnetic wave resonator. It is described (see, e.g. [4]) by rate equations for the electron population difference equation $n=n_{2}-n_{1}$ and the number of photons $N$ in the resonator:

$$
\begin{aligned}
\frac{d n}{d t}+\frac{n(t)-n_{0}}{\tau} & =I-\gamma n N \\
\frac{d N}{d t}+\sigma N & =\gamma n N
\end{aligned}
$$

where the differential gain $\gamma$, defined by $g(\omega, I)=\gamma(\omega) n(I)$, is a coefficient independent of $n$ and $\tau$ is the characteristic time describing all nonstimulated recombination processes (in high quality material, where nonradiative recombination is negligible, $\tau=\tau_{s p}$, where $\tau_{s p}$ is the characteristic time of spontaneous emission). The equilibrium population difference at 
$I=0$ is denoted by $n_{0}$. Laser generation begins when the photon gain $\gamma n$ exceeds loss $\sigma$. In this case, the stationary solution of Eq. (1) is $\gamma n=\sigma$ and $N=\left(I-I_{C}\right) / \sigma$, where $I_{C}=(\sigma / \gamma \tau)-n_{0} / \tau$.

In this simplest model, the $I$ dependence of gain $g(\omega, I)$ is parameterized by a single number $n$ and the rigidity arises automatically. Above the threshold, the mean-field equations (1) describe a wide range of phenomena, including relaxation of an arbitrary initial state to the steady state at a given $I$.

The standard STLH theory of laser linewidth is developed as follows. In the limit $N>>1$, the electromagnetic field $\tilde{E}(t)=E \exp \left(i \omega_{0} t\right)$ of a single resonator mode is considered classical, characterized by amplitude and phase. Here $\omega_{0}$ is the mode frequency, and $E$ is a complex vector that may be slowly varying in time. In the mean-field approximation, the phase $\varphi$ of the field is definite, while its amplitude is proportional to $\sqrt{N}$, i.e. $E \sim \sqrt{N} \exp (i \varphi)$. Beyond the mean-field approximation the quantities $N, n$ and $\varphi$ fluctuate in time due to the randomness of recombination and relaxation processes. It is these fluctuations that determine the linewidth in the conventional STLH approach. In an idealized laser, the fluctuations arise from randomness of spontaneous emission. All fluctuations of interest, including spontaneous emission, can be described classically in the sense that they are generated by $\delta$-correlated Langevin forces (white noise). The reason for the classical description of fluctuations is that the time scale we are interested in (of order the inverse linewidth) is long compared to all kinetic relaxation times.

In the limit $N>>1$, where the fluctuations in the number of photons are small, $\delta N<<N$, the fluctuations of $\varphi$ are decoupled from those of $n$ and $N$. Fluctuations $\delta N$ and $\delta n$ give rise to the intensity noise, while only fluctuations of the phase, $\delta \varphi$, contribute to the linewidth. These fluctuations correspond to a random walk of the complex variable $E$ of a constant modulus (see e.g. [3]). Each event of spontaneous emission adds to vector $E$ a small $\delta E \sim \sqrt{\hbar \omega_{0}}$. The angle between the two complex numbers $E$ and $\delta E$ is random and both the amplitude and the phase of the sum $E+\delta E$ are varying. The amplitude variation corresponds to $\delta N$ and, according to Eq. (1), it relaxes to its steady-state value, while $\delta \varphi \approx \delta E / E \approx 1 / \sqrt{N}$. The diffusion coefficient describing the angular random walk, $D_{\varphi}=(\delta \varphi)^{2} / \tau_{s p}$, determines the laser linewidth, $\Gamma=D_{\varphi}$, which thus turns out to be inversely proportional to the intensity of laser emission,

$$
\Gamma_{S T L H}=\frac{1}{\tau_{s p} N}
$$


Thus, at large $N$, the linewidth is much smaller than any characteristic frequency of the system, such as the spectral width of the laser cavity $\sigma(\omega)$, the rate of electronic collisions $1 / \tau_{e e}$ that determine the broadening of the quantum electronic levels in semiconductors, the spectral width of the gain $g(\omega)$, and the spontaneous emission rate $1 / \tau_{s p}$.

We would like to stress that the STLH approach essentially relies on the assumption that the mean-field equations have a singular solution with no width at all. Discussion of this assumption requires a detailed analysis of the injection-level dependence $g(\omega, I)$, which in turn requires a consideration of energy and frequency dependences of the electron and photon distributions, $n_{\varepsilon}$ and $N_{\omega}$, respectively.

We now present such a consideration based on Boltzmann's kinetic equation. It turns out that singular solutions are ruled out in the kinetic description which yields a finite laser linewidth already in the absence of fluctuations.

The simplest kinetic equation describing the energy distribution of electrons $n_{\varepsilon}$ and photons $N_{\omega}$ is of the form

$$
\begin{aligned}
\frac{d n_{\varepsilon}}{d t} & =-\gamma_{\varepsilon} n_{\varepsilon} N_{\omega}+I_{\varepsilon}+S\left\{n_{\varepsilon}\right\} \\
\frac{d N_{\omega}}{d t} & =\gamma_{\varepsilon} n_{\varepsilon} N_{\omega}-\sigma_{\omega} N_{\omega}
\end{aligned}
$$

where the energy parameters $\varepsilon$ and $\omega$ are related by $\omega=\varepsilon(k)+E_{G}$, with $E_{G}$ being the bandgap energy and $\varepsilon(k) \equiv \varepsilon_{e}(k)+\varepsilon_{h}(k)$ the kinetic energy of carriers at a wavevector $\boldsymbol{k}$ corresponding to the transition. In terms of the dimensionless $n_{\varepsilon}$, the total electron population difference $n$ that enters Eq. (1) can be expressed as

$$
n=\int_{0}^{\infty} n_{\varepsilon} \nu(\varepsilon) d \varepsilon,
$$

where $\nu(\varepsilon)$ is the density of electronic states. Similarly, $I=\int I_{\varepsilon} \nu(\varepsilon) d \varepsilon$ is the total injection level, where $I_{\varepsilon}$ is the differential injection intensity.

The collision integral $S$ has contributions from electron-electron, electronphonon interactions, and non-stimulated recombination,

$$
S\left\{n_{\varepsilon}\right\}=S_{e e}+S_{e-p h}+S_{r e c} .
$$

We consider the simplest situation when the electron-electron scattering rate $1 / \tau_{e e}$ is fastest. This situation is also most relevant for semiconductor 
lasers operating at room temperature. The collision integral $S_{e e}$ is nullified by the Fermi distribution function $n_{\varepsilon}^{F}$, parameterized by chemical potential $\mu_{\text {eff }}$ and temperature $T_{\text {eff }}$. These parameters are determined from the conservation laws for the number of particles and energy, which can be obtained from (3) by integrating over $\varepsilon$ and $\omega$. At room temperature, the energy relaxation rate is fast and one has $T_{\text {eff }}=T$.

The distribution function $n_{\varepsilon}$ deviates from the Fermi shape in a narrow interval of energies of order the linewidth $\Gamma$, where $N_{\omega} \neq 0$ and $n_{\varepsilon} \equiv n_{\varepsilon}^{F}+$ $\delta n_{\varepsilon}$. The typical energy exchange involved in electron-electron scattering events is of the order of $T$ and in the limit $\Gamma<<T$ the relaxation time approximation for electron-electron scattering is exact,

$$
n_{\varepsilon} f(\varepsilon) \equiv \sigma_{\varepsilon} / \gamma_{\varepsilon} \Gamma
$$

The reason for this is that $\delta n_{\varepsilon}$ in region $\Gamma$ is formed by incoming and outgoing fluxes from a much larger region of order $T_{\text {eff }}$ or $\mu_{\text {eff }}$ (whichever is larger). According to Eq. (3b), in a stationary state $\left(d N_{\omega} / d t=0\right)$ the electron distribution function is pinned in region $\Gamma$ and is independent of the injection level $I$ or its energy distribution $I_{\varepsilon}$ :

$$
n_{\varepsilon}=\sigma_{\omega} / \gamma_{\varepsilon}
$$

On the other hand, the electronic distribution in the region outside $\Gamma$ (where $N_{\omega}=0$ ) is not pinned because the escape rate from the outside region into the active region $\Gamma$ is finite and characterized by a time constant of order $\tau_{e e}$. The total electron concentration outside region $\Gamma$ hence grows with the injection $I$. This means that the width of $\Gamma$ itself increases with $I$.

To make this argument quantitative, we note that $\delta n_{\varepsilon}$ vanishes at the edges of region $\Gamma$. Depending on the shape of the function $f(\varepsilon)=\sigma_{\omega} / \gamma_{\varepsilon}$ in the right-hand side, Eq. (6) may have many solutions which correspond to the existence of multiple lasing modes in the mean-field approximation. Let us focus on the single-mode case, when $f(\varepsilon)$ has a single minimum at $\varepsilon=\varepsilon_{0}$ and is approximated by $f(\varepsilon)=f\left(\varepsilon_{0}\right)+a\left(\varepsilon-\varepsilon_{0}\right)^{2}$, where $f\left(\varepsilon_{0}\right) \approx 1$, see Fig. 1 . The shape of $f(\varepsilon)$ can be characterized by a halfwidth, $\Delta \approx 2 \sqrt{1 / a}$. In the case when $\sigma_{\omega}$ is a sharper function than $\gamma_{\omega}$, the quantity $\Delta$ is the resonator linewidth. Within the interval $\Gamma$ we can write

$$
\delta n_{\varepsilon}=\frac{1}{4} a \Gamma^{2}-a\left(\varepsilon-\varepsilon_{0}\right)^{2}
$$

where the constant is chosen so that $\delta n_{\varepsilon}=0$ for $\varepsilon-\varepsilon_{0}= \pm \frac{1}{2} \Gamma$. 


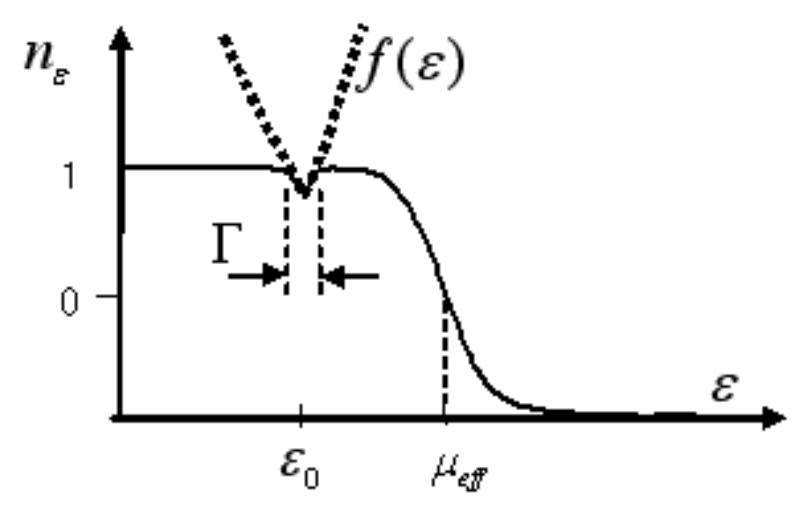

Figure 1: Schematic representation of the electron energy distribution and function $f(\varepsilon)=\sigma_{\omega} / \gamma_{\varepsilon}$. These functions coincide in region $\Gamma$.

Integrating Eq. (3a) over all energies in the stationary case $\left(d n_{\varepsilon} / d t=0\right)$ we find

$$
I-I_{C}=\int_{0}^{\infty} \sigma(\omega) N(\omega) d \omega
$$

where the threshold injection $I_{C}$ equals

$$
I_{C}=-\int_{0}^{\infty} S_{r e c} \nu(\varepsilon) d \varepsilon
$$

(terms $S_{e-p h}$ and $S_{e e}$ drop out when integrated over all energies since they conserve the number of electrons). We note that the integrand in (8) is nonvanishing only in the small region $\Gamma$ that is much narrower than either the effective temperature $T_{\text {eff }}$ or the Fermi level $\mu_{\text {eff }}$. Therefore, if we integrate Eq. (3a) over $\Gamma$, we find

$$
I-I_{C}=-\int_{\Gamma} \frac{\delta n_{\varepsilon}}{\tau_{e e}} \nu(\varepsilon) d \varepsilon .
$$

Substituting Eq. (7) into Eq. (10) we obtain an estimate of the laser linewidth:

$$
\Gamma^{3}=\frac{6}{a \nu\left(\varepsilon_{0}\right)}\left(I-I_{C}\right) \tau_{e e}
$$


or, equivalently,

$$
\Gamma=\Delta\left(\frac{3}{2 \nu\left(\varepsilon_{0}\right) \Delta}\right)^{1 / 3}\left[\left(I-I_{C}\right) \tau_{e e}\right]^{1 / 3} .
$$

We see that the linewidth in the mean-field approximation increases with pumping. This is in drastic contradiction with the conventional STLH result (2) that predicts a linewidth decreasing with $I$.

The fundamental reason for this discrepancy is the assumption by STLH of a singular, $\delta\left(\omega-\omega_{0}\right)$ like, frequency dependence of the field $E(\omega)$ in the absence of fluctuations. In contrast, the solutions of kinetic equations are smooth functions of $\varepsilon$ and $\omega$ and do not exhibit any singularity. Consequently, an account of fluctuations would make only a small correction to our result.

It should be cautioned that validity of kinetic equations (3) requires that the uncertainty in electronic energies due to collisions be smaller than the interval of electronic energies that we are interested in $\left(1 / \tau_{e e}<<\Gamma\right)$. According to Eq. (11), this condition is satisfied at sufficiently high injection intensities. However, semiconductor lasers at room temperature are typically in the opposite regime $1 / \tau_{e e}>>\Gamma$. In this regime we are concerned with the details of the electron distribution function resolved on a much finer scale than that on which the single electronic states themselves are well defined. We are not aware of any example in kinetic theory where a quantitative description of such a situation has been developed.

Available experiments lend conclusive support neither to our result nor STLH. At low intensities above threshold one observes a decreasing linewidth but at higher intensities the linewidth often saturates and then re-broadens, so that $\Gamma(I)$ exhibits a minimum (see, e.g., Fig. 6.15 in [5], Fig. 9.11 in [6], or the more recent data [7]). One of the possible scenarios that would reconcile the two pictures is that at low injection, the mean-field linewidth given by the kinetic equation approach happens to be much smaller than the STLH linewidth given by (2), i.e. $\Gamma(I)<\Gamma_{S T L H}(I)$ at least near threshold. In this case, the initial decrease of the linewidth with $I$ could be attributed to a STLH-like mechanism, whereas for larger $I$ the increasing mean-field linewidth takes over and one has re-broadening.

In the opposite limit, which we find more realistic, there is no range for STLH to hold and we would have to conclude that the decreasing linewidth lacks theoretical explanation. Development of a satisfactory linewidth theory would then require inclusion of additional phenomena that go beyond the kinetic description. 
We see that the standard theory of laser linewidth is unsatisfactory. The theory attributes the spectral width of laser oscillation to fluctuations brought about by random spontaneous emission events and is essentially based on the assumption that in the absence of fluctuation laser radiation is monochromatic. We have shown that this assumption is inconsistent and that already in the mean field model the laser oscillations have a finite spectral linewidth that furthermore increases with pumping.

Our consideration was restricted to semiconductor lasers but our conclusion is likely to be more general, applicable to other lasers as well, such as solid-state lasers and gas lasers. The question of why the laser linewidth can be much narrower than either the gain spectrum or the resonator linewidth is begging a theoretical explanation.

We are grateful to R.F. Kazarinov for useful discussions.

\section{References}

[1] A.L. Schawlow and C.H. Townes, Phys. Rev. 112, 1940 (1958).

[2] M. Lax, Phys. Rev. 160, 290 (1967); R.D. Hempstead and M. Lax, Phys. Rev. 161, 350 (1967).

[3] C.H. Henry, IEEE J. Quantum Electronics QE-18, 259 (1982); ibid. QE-19, pp. 1391-1397 (1983).

[4] A.E. Siegman, Lasers (University Science Books, Sausalito, CA, 1986).

[5] G.P. Agrawal and N.K. Dutta, Semiconductor Lasers, 2-nd ed. (Van Nostrand, New York, 1993).

[6] G. Morthier and P. Vankwikelberge, Handbook of Distributed Feedback Laser Diodes (Artech House, Boston, 1997).

[7] H. Su, L. Zhang, R. Wang, T.C. Newell, A.L. Gray, and L.F. Lester, IEEE Phot. Technol. Lett. 16, 2206 (2004). 